\title{
Shoulder assessment by smartphone: a valid alternative for times of social distancing
}

\author{
Alon Rabin ${ }^{1} \cdot$ Oleg Dolkart ${ }^{2} \cdot$ Efi Kazum $^{2} \cdot$ Reut Wengier $^{2} \cdot$ Yariv Goldstein $^{3} \cdot$ Eran Maman $^{2} \cdot$ Ofir Chechik $^{2}$
}

Received: 16 August 2020 / Accepted: 1 January 2021 / Published online: 13 January 2021

(c) The Author(s), under exclusive licence to Springer-Verlag GmbH, DE part of Springer Nature 2021

\begin{abstract}
Background The delivery of orthopaedic care via telemedicine services has the potential to promote accessibility and decrease medical care expenses, while facilitating the control of infectious disease spreading. The purpose of this study was to assess agreement regarding diagnosis, recommended course of management and the perceived need for additional diagnostic testing between a video examination (VE) and a face to face (FTF) assessment of patients with shoulder disorders. Methods Forty-seven (18 females) patients presenting to a shoulder surgery clinic were assessed consecutively by VE and a FTF examination. All assessments were conducted by a shoulder specialist. Agreement regarding the established diagnosis, the recommended course of management and the need for additional diagnostic tests was assessed using percent agreement and kappa $(95 \% \mathrm{CI})$ coefficient. Differences in the content, duration and satisfaction between the two examination modes were also assessed.

Results Percent agreement and kappa (95\% CI) coefficient for agreement regarding diagnosis were $85.1 \%$ and $0.82(0.69$ 0.94), respectively. Percent agreement and kappa (95\% CI) coefficient regarding the recommended course of management and the need for additional diagnostic testing were $61.7 \%$ and $0.43(0.22-0.63)$, and $74.5 \%$ and $0.49(0.25-0.74)$, respectively. The VE resulted in collection of less physical examination information, took longer to complete and was associated with less satisfaction by both patient and examiner.

Conclusions Video examination of patients with shoulder disorders may present a valid alternative to FTF examination. Nevertheless, the content of the video-based physical examination may need to be modified to facilitate a clearer detection of indications for specific interventions or diagnostic tests.
\end{abstract}

Keywords Telemedicine $\cdot$ Shoulder $\cdot$ Physical examination $\cdot$ Social distancing

\section{Introduction}

Telemedicine, the means of providing medical services to individuals in remote locations by way of video examination (VE) has been proposed as a means to shorten waiting periods, reduce medical care expenditures, and facilitate the delivery of medical services to populations with more limited access [1,2]. The coronavirus disease 2019 (COVID

Oleg Dolkart

dolkarto@gmail.com

1 Department of Physiotherapy, Ariel University, Ariel, Israel

2 Division of Orthopaedic Surgery, Tel Aviv Sourasky Medical Center, Sackler Faculty of Medicine, Tel Aviv University, 6 Weizman Street, 6423906 Tel Aviv, Israel

3 Assuta Ashdod Meical Center, Ashdod, Israel
19) pandemic has dramatically highlighted another unique advantage of VE, that is, the ability to allow timely delivery of valuable medical services while maintaining social distancing policies and minimizing the risk of widespread contamination of infectious diseases.

The literature concerning the use of telemedicine services in orthopaedic practice suggests this mode of delivery may be feasible, cost-effective, and acceptable to both provider and recipient [2-7]. One aspect that has received much less attention in the available literature is the effect of a VE on clinical decision making related to orthopaedic care. Thus far, studies from the field of rehabilitation suggest some aspects of a video-based physical examination can be performed with acceptable reliability and validity [8]. Furthermore, a high level of agreement regarding patient diagnosis and management has been demonstrated between a standard assessment by a hand surgeon and a surgeon presented with 
digital images of the physical examination and radiographs of the same patient [9]. Nevertheless, the level of agreement concerning patient diagnosis and the recommended course of management between real-time independent VE and FTF examinations has not been previously determined. Given the importance of these attributes to the implementation of telemedicine orthopaedic practice, the aim of this study was to assess the between-examiner agreement regarding diagnosis, the recommended course of management, and the perceived need for conducting additional diagnostic testing following a standard (FTF) versus a VE among patients with shoulder disorders. The secondary goal of the study was to describe and compare the characteristics of either examination mode (FTF versus VE), as well as the satisfaction of the examiner and patient with either procedure.

\section{Methods}

\section{Patients}

Consecutive patients referred to an outpatient shoulder clinic within a large metropolitan medical center were recruited for the study. Inclusion criteria were age 18 years or older, having a unilateral shoulder complaint, as well as possessing a cellular phone with a video call application. Potential patients were excluded if they did not possess good verbal communication skills. The study was approved by the Institutional Review Board, and all patients read and signed an informed consent form prior to participation.

\section{Examiners}

Two board-certified and fellowship-trained orthopaedic surgeons performed all data collection for this study. Prior to data collection the two surgeons met for $4 \mathrm{~h}$ to discuss and standardize all procedures for the study. Subsequently, pilot testing of five randomly selected patients attending the outpatient clinic was performed. Data from these five patients were not included in the primary analysis.

\section{Procedure}

After providing informed consent each patient underwent a standard (FTF) examination by one examiner as well as a VE by another examiner. The examiner performing the FTF examination was the surgeon originally designated to see the patient during his/her scheduled visit to the outpatient clinic. Consequently, the order of examinations (FTF versus VE) was based on examiner availability. For example, when the examiner originally scheduled to see the patient was having long waiting times, the patient was offered to undergo a VE first, in an attempt to minimize the total waiting time spent in the clinic.

\section{Standard (FTF) examination}

The FTF examination included collection of demographic information, history, physical examination and review of pertinent imaging as per usual practice. At the completion of the examination, the examiner established a diagnosis, a recommended course of management, and the need for additional diagnostic studies. The amount of time required to complete the examination was documented and the patient as well as the examiner completed a 5-item questionnaire regarding their satisfaction with the standard examination. Each item was scored using a numeric 6-point (0-5) scale with higher scores indicating greater satisfaction (Appendix 1).

\section{Video examination}

The examiner used his cell phone to call the patient who was situated in a remote examination room. The patient held his/ her cell phone during the history portion of the examination, while his/her companion (or a research assistant in the absence of a companion) handled the cell phone during the physical examination. The examiner guided the companion/ research assistant in positioning the cell phone, zooming in or zooming out to obtain an optimal view of the patient.

The content of the history was similar to that of the FTF examination. For the physical examination, the patient was first asked to stand up while the companion was guided to position the cell phone so that the examiner could get a clear view of both shoulders from the front, the side, and the back. The front view was used to assess for any sternoclavicular or acromioclavicular joint deformity, pectorals, deltoid, and biceps brachi muscle mass. The side view was used to assess thoracic kyphosis and deltoid muscle mass, while the back view was used to assess for scapulothoracic alignment, supraspinatus, and infraspinatus muscle mass. Active and passive range of motion $(\mathrm{A} / \mathrm{PROM})$ were assessed from a side view (forward flexion), a front view (frontal-plane abduction and external rotation), and a back view (internal rotation behind the back) [10]. Through all motions, AROM was assessed first followed, when necessary, by PROM using the patient's uninvolved hand. Examiner continued by instructing the patient in performing resisted strength tests, special tests, and self-palpation as was deemed necessary by the examiner. Strength (using the uninvolved hand or a nearby object such as a door or table) as well as any special tests and self-palpation necessary to establish a diagnosis $[10,11]$. This was accomplished by guiding the patient to use his/her uninvolved hand to provide resistance or to palpate anatomical landmarks, or position the shoulder into a 
required position through the use of an available external object. For example, instead of passively positioning the shoulder into flexion and internal rotation as in the Hawkings test, the patients was asked to place his/her the involved hand on the opposite shoulder and then bring the elbow up to chest level. Likewise, instead of providing manual resistance to perform the Jobe, Speed, or O'brian test, the patients was guided to provide self-resistance with his/her uninvolved hand after placing the involved shoulder in the appropriate test position. Similarly, instead of placing the shoulder into the anterior apprehension position, the patients was guided to hook the involved forearm on the side of a door with the shoulder at $90^{\circ}$ of abduction and then turn his/her trunk away to replicate the anterior apprehension position. Following the physical examination, the patient was asked to read the written reports associated with any previously performed diagnostic studies, or to present the report for review by the examiner through the cell phone screen.

At the conclusion of all assessment procedures, the examiner established a diagnosis, a recommended course of management, and the need for additional diagnostic studies. The amount of time required to complete the VE was documented and the patient as well as the completed another 5-item questionnaire (Appendix 1) regarding their satisfaction with the VE procedure.

\section{Outcome measures}

The diagnosis, recommended course of management, and the need for additional diagnostic studies served as the primary outcome measures for the study. Diagnosis was classified into eight distinct subgroups: none (no diagnosis could be established), rotator cuff disease, shoulder instability, superior labrum anterior posterior (SLAP) lesions, adhesive capsulitis, post-operative follow-up, shoulder fracture, glenohumeral osteoarthritis, or other (i.e. long head of biceps lesions, acromioclavicular joint sprain, etc.). Intervention was classified into one of four options: none (no intervention recommended), physical therapy, subacromial injection, or surgery. The need for additional diagnostic studies was classified as present or absent.

The content of the standard and video-based examination, the amount of time required to complete either examination, as well as the satisfaction of the examiner and patient with each examination mode served as secondary outcome measures.

\section{Analyses}

Data was summarized using descriptive statistics with measures of central tendency and dispersion for continuous variables and frequency counts for categorical variables. For the primary purpose of the study percent agreement and kappa (к) coefficient $(95 \% \mathrm{CI})$ for the between-examiner agreement regarding diagnosis, management, and the need for additional diagnostic studies were calculated. A prevalenceadjusted kappa was used to describe the agreement between examiners regarding the need for additional diagnostic studies because of a disproportioned agreement on negative tests (i.e. lack of need) versus positive tests ( 32 versus 3 cases) [12]. In addition differences in the frequency of use of the various management options, as well as in the frequency of referral for additional diagnostic tests were compared using separate McNemar tests.

For the secondary purpose of the study differences in the characteristics of the either examination, as well as differences in the satisfaction of the examiner and patient with either examination mode were compared using separate paired $T$ tests and McNemar tests for interval and categorical variables, respectively. All analyses were performed using SPSS version 25 , with an a priori $P$ value of 0.05 .

\section{Results}

Forty-seven (18 females) patients were recruited for the study. Table 1 summarizes the main demographic and clinical characteristics of the sample. Eighteen (38.3\%) patients were seen as part of their post-operative follow-up, and four (8.5\%) additional patients were seen for follow-up following

Table 1 Mean \pm SD demographic, history, and clinical characteristics of the sample

\begin{tabular}{ll}
\hline Variable & Value \\
\hline Sex, $n(\%)$ female & $18(36.0)$ \\
Age, years & $44.6 \pm 22.0$ \\
Height, cm & $172.3 \pm 10.4$ \\
Weight, kg & $75.0 \pm 13.7$ \\
Dominant side, $n(\%)$ right & $42(84.0)$ \\
Dominant side involved, $n(\%)$ & $26(52.0)$ \\
Duration of symptoms (months) & $29.3 \pm 81.7$ \\
Diagnosis, $n(\%) *$ & \\
Post-operative follow-up & $17(36.2)$ \\
RCD & $9(19.1)$ \\
SLAP lesion & $7(14.9)$ \\
Shoulder instability & $5(10.6)$ \\
Shoulder girdle fracture & $4(8.5)$ \\
Glenohumeral OA & $1(2.1)$ \\
Adhesive capsulitis & $1(2.1)$ \\
Other & $2(4.2)$ \\
First examination, $n(\%)$ FTF & $35(70.0)$ \\
\hline
\end{tabular}

$O A$ osteoarthritis, $R C D$ rotator cuff disease, SLAP superior labrum anterior posterior

*Diagnoses based on standard examination 
a non-operatively managed fracture. The remaining 25 $(53.2 \%)$ patients were seen for a variety of shoulder-related complaints (i.e. pain, stiffness, instability). The FTF examination was completed on all 47 patients, while the VE was terminated prematurely in 3 patients. In two cases, this was due to a poor sound quality and in one additional case the patient's cell phone battery ran out.

Percent agreement and kappa coefficient (95\% CI) for inter-examiner agreement regarding diagnosis were $85.1 \%$ and $0.82(0.69-0.94)$, respectively. Percent agreement and kappa coefficient $(95 \% \mathrm{CI})$ regarding the selected course of management and the need for additional diagnostic testing were $61.7 \%$ and $0.43(0.22-0.63)$, and $74.5 \%$ and 0.49 (0.25-0.74), respectively.

Table 2 details each examiner's selection of the different management options and the need for additional diagnostic testing. The frequency of all selected management options and for the need of additional diagnostic tests did not differ between examiners $(P \geq 0.09)$.

Table 3 outlines the characteristics of the FTF and the VE. Significantly less use of observation $(82.2 \%$ versus $95.7 \%, P=0.01)$ and PROM tests $(13.3 \%$ versus $34.0 \%$, $P=0.02)$ were noted during the VE. The duration of the VE was significantly longer (12.4 min versus $9.3 \mathrm{~min}, P<0.01$ ).

Table 4 presents examiner and patient satisfaction with the different aspects of each examination. Both examiner and patient were significantly less satisfied with each aspect, as well as the entire experience of the VE.

\section{Discussion}

A substantial to almost perfect agreement regarding the diagnosis of patients with various shoulder disorders was demonstrated between an assessment performed with the patient present in the examination room and an assessment performed by way of a video call. Agreement concerning the course of management and the need for additional diagnostic studies was moderate. Our findings compare favourably with previous investigations into the inter-rater reliability of the diagnosis of patients with shoulder complaints during
Table 3 Differences in the components of the FTF and video assessment

\begin{tabular}{|c|c|c|c|}
\hline Variable & $\begin{array}{l}\text { Face-to- } \\
\text { face assess- } \\
\text { ment }\end{array}$ & Video assessment & $P$ value \\
\hline $\begin{array}{l}\text { Complete assessment, } n \\
(\%)\end{array}$ & 47 & 44 & NA \\
\hline Complete history, $n(\%)$ & 47 & 46 & NA \\
\hline Complete PE, $n(\%)$ & 47 & 44 & NA \\
\hline PE-observation, $n(\%)$ & $45(95.7)$ & $37(82.2)$ & 0.01 \\
\hline $\mathrm{PE}-\mathrm{AROM}, n(\%)$ & $44(93.6)$ & $41(91.1)$ & 0.24 \\
\hline PE—PROM, n (\%) & $16(34.0)$ & $6(13.3)$ & 0.02 \\
\hline PE-MMT, $n(\%)$ & $22(46.8)$ & $16(35.6)$ & 0.08 \\
\hline PE—special tests, $n(\%)$ & $18(38.3)$ & $13(29.5)$ & 0.36 \\
\hline PE—no. of special tests, $n$ & $0.68 \pm 1.1$ & $0.34 \pm 0.60$ & 0.07 \\
\hline PE-palpation, $n(\%)$ & $13(27.7)$ & $11(25.0)$ & 0.79 \\
\hline $\begin{array}{l}\mathrm{PE} \text {-no. of palpation } \\
\text { points }\end{array}$ & $17(36.2)$ & $13(28.9)$ & 0.44 \\
\hline Total duration, minutes & $9.3 \pm 3.8$ & $12.4 \pm 3.9$ & $<0.01$ \\
\hline
\end{tabular}

$A R O M$ active range of motion, $M M T$ manual muscle testing, $P E$ physical examination, PROM passive range of motion

independent assessments of patients that are physically present within the examination room [13-17]. Only one previous study [16] reported a better level of agreement than the one reported in the current investigation. These findings suggest the use of a video call for examining patients in remote locations may not considerably alter examiner's impression of a diagnosis and may, therefore, serve a valid alternative to the standard assessment when the physical presence of a patient is contra-indicated or involves considerable time and/ or travel expenses.

The overall frequency of use of specific interventions and the overall perceived need for additional diagnostic tests did not differ between the two examination modes. Although this implies no major change in practice patterns as a result of the use of a VE, the level of agreement regarding selected interventions and the need for additional diagnostic tests was only moderate. While some of these differences may be due to differences in individual examiner preferences, it is

Table 2 Frequencies and level of agreement between examiners on the course of management and the need for additional diagnostic tests

\begin{tabular}{lccccc}
\hline & Face-to-face & Video examination & $P$ value & Percent agreement & Kappa (95\% CI) \\
\hline No intervention, $n(\%)$ & $9(19.1)$ & $16(34.0)$ & 0.09 & $61.7 \%$ & $0.43(0.22-0.63)$ \\
Physical therapy, $n(\%)$ & $24(51.1)$ & $21(44.7)$ & 0.60 & & \\
Subacromial injection, $n(\%)$ & $4(8.5)$ & $2(4.3)$ & 0.48 & & \\
Surgery, $n(\%)$ & $10(21.3)$ & $8(17.0)$ & 0.68 & & \\
Additional diagnostic tests, $n(\%) *$ & $11(23.4)$ & $8(17.0)$ & 0.55 & $74.5 \%$ & $0.49(0.25-0.74)$ \\
\hline
\end{tabular}

*Prevalence adjusted kappa 
Table 4 Patient and examiner satisfaction

\begin{tabular}{lccc}
\hline Variable & Standard assessment & Video assessment & P value \\
\hline Examiner & & & \\
Ability to obtain medical history & $5.0 \pm 0.0$ & $4.4 \pm 0.9$ & $<0.01$ \\
Ability to perform physical examination & $5.0 \pm 0.0$ & $3.6 \pm 1.1$ & $<0.01$ \\
Ability to view imaging studies & $5.0 \pm 0.0$ & $4.3 \pm 0.8$ & $<0.01$ \\
Ability to establish diagnosis and treatment plan & $5.0 \pm 0.0$ & $4.6 \pm 0.6$ & $<0.01$ \\
Ability to explain diagnosis and plan and to & $5.0 \pm 0.0$ & $4.6 \pm 0.9$ & $<0.01$ \\
$\quad$ address questions & & & $<0.01$ \\
$\quad$ Total examiner satisfaction score & $25.0 \pm 0.0$ & $21.0 \pm 3.6$ & $<0.01$ \\
Patient & & & $<0.01$ \\
Ability to communicate with examiner & $5.0 \pm 0.0$ & $4.4 \pm 0.8$ & $<0.01$ \\
Ability to ask questions & $5.0 \pm 0.0$ & $4.5 \pm 0.9$ & $<0.01$ \\
Lost important information & $4.9 \pm 0.6$ & $4.3 \pm 0.9$ & $<0.01$ \\
Understanding diagnosis and treatment plan & $5.0 \pm 0.0$ & $4.7 \pm 0.6$ & $<0.01$ \\
Response to questions and concerns & $5.0 \pm 0.0$ & $4.7 \pm 0.6$ & $22.6 \pm 2.8$ \\
$\quad$ Total patient satisfaction score & $24.9 \pm 0.6$ & & \\
\hline
\end{tabular}

also possible that they resulted from differences in the quantity and/or quality of information available to the examiner following each respective examination. For the most part examination by video was performed using fewer physical manoeuvres. While this may not have affected the ability to reach a diagnosis, the more limited information available to the examiner following the VE may have altered his impression regarding the need for specific interventions such as physical therapy and/or subacromial injection. For example, as PROM and MMT were performed less frequently during the VE, indications for physical therapy such as limited PROM and/or muscle weakness may have been missed. Post hoc analysis revealed an especially low level of agreement between the examiners regarding the need for physical therapy (percent agreement: 59.1\%, kappa coefficient: 0.17 ). Likewise, potential indicators for the need of a subacromial injection such as a positive Hawkins or Neer test may have been more difficult to obtain during the VE and, conversely, contra-indications to subacromial injections such as considerable strength deficits may also have been more difficult to obtain by way of a VE. Consequently, the examiner performing the VE may have been more reluctant to offer a subaromial injection (two versus four injections following the FTF examination).

The advantages of assessing patients from remote locations are evident in terms of economic costs, patient convenience and infection control [18]. However, these advantages can only be realized when the process of assessment by video results in similar outcomes to the standard of care. In recently published study by Rizzi et al. [19], patients and orthopaedic surgeons documented high levels of satisfaction with telehealth encounters during the novel coronavirus (COVID-19) pandemic. Telemedicine does not appear to be a replacement for all in-person clinic encounters, however, when used in the appropriate context demonstrated favourable results. Our study is the first to compare between the outcomes and characteristics of the two assessment modes and demonstrates a high level of agreement regarding diagnosis, and a moderate level of agreement regarding the selection of intervention and the need for additional diagnostic testing. These findings suggest that assessment of patients with shoulder disorders by way of a VE may serve an acceptable alternative to a FTF examination. Alongside this finding, our study also suggests the need to refine the VE and, in particular, the contents of the physical examination, to facilitate the recognition of indications for commonly prescribed interventions. Perhaps a shift toward an assessment of functional ability, as opposed to measures of physical impairments may help circumvent some of the pitfalls of the VE. Several standardized physical performance measures already exist for assessing the functional ability of patients with several operative and non-operative shoulder conditions [20, 21]. As these measures focus more on the ability to perform basic or more advanced activities of daily living they seem more suited for administration by way of a VE, and may, therefore, help to more clearly establish the need for interventions such as physical therapy or subacromial injection.

Our study has several important limitations. First, the study was performed within a tertiary care unit operated by highly specialized shoulder surgeons. Consequently, our findings may not be generalizable to a primary care or even a more general orthopaedic setting. Second, our sample was comprised of a mixed group of patients including non-operative, post-operative, and patients following shoulder-related fractures. We acknowledge that the decision-making process regarding diagnosis, course of management, and the need for additional diagnostic tests differs considerably among these 
patient populations. It is possible that dividing the sample into more homogenous subgroups would have resulted in different levels of agreement; however, given the preliminary nature of this study, we preferred to assess inter-examiner agreement among the entire of scope of patients visiting a shoulder surgery clinic. Given that some of the participants were seen for follow-up examination, it is acknowledged that the surgeon performing the FTF was already familiar with their diagnosis and the course of management. Finally, as some patients were not accompanied by a companion during their visit to clinic, we used a research assistant for handling the patient's cell phone during the VE. This may have artificially facilitated the communication with the examiner, possibly leading to more optimal examination conditions. However, given that we explicitly instructed the research assistant to simply follow the examiner's requests during the VE, we believe the effect of this limitation was minimal.

\section{Conclusions}

A VE of patients visiting a shoulder surgery clinic may serve a valid alternative to a standard examination given the high level of agreement regarding patient diagnosis and the moderate level of agreement regarding the course of management and need for additional diagnostic testing. Nevertheless, as the VE seems to allow for a less complete physical examination, alternative tests may need to be developed to facilitate a more informed decision regarding the need for specific interventions or diagnostic inquiries.

Funding None.

\section{Compliance with ethical standards}

Conflict of interest None.

Ethical approval All procedures were approved by the Institutional Animal Care and Use Committee.

\section{References}

1. Blank E, Lappan C, Belmont PJ Jr, Machen MS, Ficke J, Pope R, Owens BD (2011) Early analysis of the United States Army's telemedicine orthopaedic consultation program. J Surg Orthop Adv 20(1):50-55

2. Rowell PD, Pincus P, White M, Smith AC (2014) Telehealth in paediatric orthopaedic surgery in Queensland: a 10-year review. ANZ J Surg 84(12):955-959. https://doi.org/10.1111/ans.12753

3. Haukipuro K, Ohinmaa A, Winblad I, Linden T, Vuolio S (2000) The feasibility of telemedicine for orthopaedic outpatient clinics - a randomized controlled trial. J Telemed Telecare 6(4):193198. https://doi.org/10.1258/1357633001935347
4. McGill A, North J (2012) An analysis of an ongoing trial of rural videoconference fracture clinics. J Telemed Telecare 18(8):470-472. https://doi.org/10.1258/jtt.2012.GTH110

5. Tachakra S, Hollingdale J, Uche CU (2001) Evaluation of telemedical orthopaedic specialty support to a minor accident and treatment service. J Telemed Telecare 7(1):27-31. https://doi. org/10.1258/1357633011936101

6. Waterman BR, Laughlin MD, Belmont PJ Jr, Schoenfeld AJ, Pallis MP (2014) Enhanced casualty care from a Global Military Orthopaedic Teleconsultation Program. Injury 45(11):17361740. https://doi.org/10.1016/j.injury.2014.03.012

7. Siow MY, Walker JT, Britt E, Kozy JP, Zanzucchi A, Girard PJ, Schwartz AK, Kent WT (2020) What was the change in telehealth usage and proportion of no-show visits for an orthopaedic trauma clinic during the COVID-19 pandemic? Clin Orthop Relat Res 478(10):2257-2263. https://doi.org/10.1097/ CORR.0000000000001396

8. Mani S, Sharma S, Omar B, Paungmali A, Joseph L (2017) Validity and reliability of Internet-based physiotherapy assessment for musculoskeletal disorders: a systematic review. J Telemed Telecare 23(3):379-391. https://doi.org/10.1177/13576 33X16642369

9. Abboud JA, Bozentka DJ, Beredjiklian PK (2005) Telemedicine consultation for patients with upper extremity disorders is reliable. Clin Orthop Relat Res 435:250-257. https://doi.org/10.1097/01. blo.0000155009.77961.5c

10. Tanaka MJ, Oh LS, Martin SD, Berkson EM (2020) Telemedicine in the Era of COVID-19: The virtual orthopaedic examination. J Bone Joint Surg Am 102(12):e57. https://doi.org/10.2106/ JBJS.20.00609

11. Laskowski ER, Johnson SE, Shelerud RA, Lee JA, Rabatin AE, Driscoll SW, Moore BJ, Wainberg MC, Terzic CM (2020) The Telemedicine musculoskeletal examination. Mayo Clin Proc 95(8):1715-1731. https://doi.org/10.1016/j.mayocp.2020.05.026

12. Sim J, Wright CC (2005) The kappa statistic in reliability studies: use, interpretation, and sample size requirements. Phys Ther 85(3):257-268

13. Carter T, Hall H, McIntosh G, Murphy J, MacDougall J, Boyle C (2012) Intertester reliability of a classification system for shoulder pain. Physiotherapy 98(1):40-46. https://doi.org/10.1016/j.physi o.2010.12.003

14. de Winter AF, Jans MP, Scholten RJ, Deville W, van Schaardenburg D, Bouter LM (1999) Diagnostic classification of shoulder disorders: interobserver agreement and determinants of disagreement. Ann Rheum Dis 58(5):272-277. https://doi.org/10.1136/ $\operatorname{ard}$.58.5.272

15. Hanchard NC, Howe TE, Gilbert MM (2005) Diagnosis of shoulder pain by history and selective tissue tension: agreement between assessors. J Orthop Sports Phys Ther 35(3):147-153. https://doi.org/10.2519/jospt.2005.35.3.147

16. Pellecchia GL, Paolino J, Connell J (1996) Intertester reliability of the cyriax evaluation in assessing patients with shoulder pain. J Orthop Sports Phys Ther 23(1):34-38. https://doi.org/10.2519/ jospt.1996.23.1.34

17. Storheil B, Klouman E, Holmvik S, Emaus N, Fleten N (2016) Intertester reliability of shoulder complaints diagnoses in primary health care. Scand J Prim Health Care 34(3):224-231. https://doi. org/10.1080/02813432.2016.1207139

18. Haider Z, Aweid B, Subramanian P, Iranpour F (2020) Telemedicine in orthopaedics and its potential applications during COVID19 and beyond: aa systematic review. J Telemed Telecare. https:// doi.org/10.1177/1357633X20938241

19. Rizzi AM, Polachek WS, Dulas M, Strelzow JA, Hynes KK (2020) The new "normal": Rapid adoption of telemedicine in orthopaedics during the COVID-19 pandemic. Injury. https://doi. org/10.1016/j.injury.2020.09.009 
20. Dupeyron A, Gelis A, Sablayrolles P, Bousquet PJ, Julia M, Herisson C, Pelissier J, Codine P (2010) Heterogeneous assessment of shoulder disorders: validation of the Standardized Index of Shoulder Function. J Rehabil Med 42(10):967-972. https://doi. org/10.2340/16501977-0627

21. van de Water AT, Davidson M, Shields N, Evans MC, Taylor NF (2015) The Shoulder Function Index (SFInX): a clinicianobserved outcome measure for people with a proximal humeral fracture. BMC Musculoskelet Disord 16:31. https://doi. org/10.1186/s12891-015-0481-X

Publisher's Note Springer Nature remains neutral with regard to jurisdictional claims in published maps and institutional affiliations. 